\title{
LA SIMBIOSIS INDUSTRIAL un ensayo dialógico
}

\author{
Jesús Rodarte Dávila \\ Jorge A. Salas Plata Mendoza \\ Universidad Autónoma \\ de Ciudad Juárez
}

RECIBIDO: 12 DE FEBRERO DE 2015

ACEPTAdo: 20 DE ABRIL DE 2015

\section{RESUMEN}

T a inevitabilidad de la generación de residuos y la necesidad de su

disposición, reciclado, reúso o preservación demandan la implementación de estrategias tendientes a mejorar y preservar el medio ambiente mediante la implementación de sistemas que coadyuven en este proceso como la simbiosis industrial, a través de la cual se puede obtener una economía sustentable y cuyo éxito recae en la ampliación de nuestro conocimiento acerca de cómo y por qué la gente elige interactuar entre sí de una $\mathrm{u}$ otra manera y cómo sus decisiones colectivas afectan nuestra biósfera y sus ecosistemas al paso del tiempo.

El presente trabajo parte de una comprensiva revisión de literatura mediante el análisis de artículos científicos, cuyo propósito es contrastar las posturas a favor de la simbiosis industrial y aquellas que plantean serias dudas de su aplicación, así como la conciliación entre ambas más allá de una simple formulación ecléctica.

Palabras clave: simbiosis industrial, medio ambiente, formulación ecléctica.

\section{Abstract}

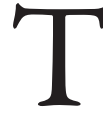

The inevitability of the generation of waste and the need for its disposal, recycling, reuse or preservation require the implementation of strategies aimed at improving and preserving the environment through the implementation of systems that contribute to this process as the industrial symbiosis through which a sustainable economy can be obtained and whose success lies in the expansion of our knowledge about how and why people choose to interact with each other and how their collecti- 
ve decisions affect our biosphere and its ecosystems to the passage of time.

This work is based on a comprehensive review of literature through the analysis of scientific articles, whose purpose is to contrast the positions in favor of the industrial symbiosis and those that raise serious questions of its application as well as the reconciliation between them beyond a simple eclectic formulation.

Keywords: industrial symbiosis, environment, eclectic formulation.

\section{INTRODUCCIÓN}

$\mathrm{L}$ a reducción de costos en la producción no debe basarse, como regla, en los despidos de trabajadores, sino, entre otras cosas, en la búsqueda de estrategias que permitan el desarrollo de economías, para evitar consumos excesivos de recursos que mejoren la competitividad y los beneficios netos (Andersen, 2007). Se trata de buscar medios para una reducción viable del consumo de materiales vírgenes mejorando la impresión de la huella ecológica (medida estandarizada de la demanda de recursos naturales contrastada con la capacidad de regeneración ecológica del planeta).

Las industrias que promueven la ecología industrial buscan cerrar el ciclo de materia y energía, y por tanto, obtener un nivel cero de residuos. Uno de los caminos para el logro de estos objetivos, es a través de la simbiosis industrial (SI) (Perry, \& Boon, 2004).

La SI se sirve de métodos que contribuyen a disminuir el impacto ambiental, mejorar la eficiencia y aumentar la rentabilidad industrial, siempre tendiendo hacia la sustentabilidad (Cote, \& Cohen-Rosenthal, 1998). Sin embargo, no todos coinciden en que la SI tenga tal potencial, ya que entran en juego aspectos culturales, la inevitabilidad de las in- eficiencias y la fragilidad de los entornos industriales. Para tal efecto, la SI abarca precisamente toda esta pirámide, ya que parte de la inevitabilidad de la generación de residuos y la necesidad de su disposición, reciclado, reúso o preservación.

\section{JERARQUía DE RESIDUOS}

$\mathrm{L}$

a jerarquía de residuos y la disposición de los mismos, es la base de la pirámide de sustentabilidad (Andersen, 2007) y para poder lograr una buena preservación de los recursos naturales, es necesario valorizar energéticamente la recuperación y el reciclaje de los residuos (Sagoff, 1998), que generalmente establecen un orden de prioridades para la mejor opción ambiental y la administración adecuada de los bienes de uso como la energía, calor, agua u otros residuos (Perry, \& Boon, 2004).

La mala eficiencia en la gestión ambiental, la planificación y el uso de los recursos conduce a una inversión expansiva en capacidad y ampliación de instalaciones de servicios públicos (plantas tratadoras de agua, combustión, caminos para el transporte, etcétera) basadas en tecnologías "viejas", que contribuyen al calentamiento global y agudizan la escasez de bienes naturales (Roberts, 2004).

\section{Simbiosis INDUSTRIAL}

$\mathrm{L}$ a experiencia de Kalundborg, Dinamarca, en la década de los enta, en la que varias empresas iniciaron un trabajo colectivo para el intercambio de materia residual y energía excedente, acotó el uso indiscriminado de los recursos naturales y contribuyó al crecimiento del desarrollo futuro de la economía de la región (Tudor, Adam, \&
Bates, 2007). El Parque de Kalundborg es el ejemplo más claro utilizado como modelo de SI en Dinamarca (Andersen, 2007), mismo que fue construido en la década de los sesenta y no fue creado expresamente como un parque industrial.

La SI se basa, principalmente, en dos elementos clave, a saber: la ecología industrial (EI) y la ecología biológica (EB) (Tudor et al., 2007). El tema de la EI es relativamente nuevo, habiendo surgido a finales de 1980. Cohen-Rosenthal (2004) hace notar que la EI se refiere principalmente a generar el menor daño en sistemas industriales y ecológicos, a través de la óptima circulación de materiales y energía. El aprendizaje colectivo puede fomentar socios industriales potenciales y gradualmente cooperar en el intercambio de subproductos y/o compartir infraestructura común por su proximidad (Cote, \& Cohen-Rosenthal, 1998). Este tipo de coevolución de aprendizaje, con la ayuda de modelos participativos, podría ayudar a fomentar la SI. El campo más importante de la SI, se encuentra en los ecosistemas biológicos (Norton, Constanza, \& Bishop, 1998), la cual busca imitar estos ecosistemas donde se incorporan productos "basura" de un proceso a otro como la utilización del dióxido de carbono y el oxígeno en los procesos de fotosíntesis y respiración (Chiu, \& Yong, 2004). La SI destaca la importancia del aprendizaje de los sistemas de descomposición y reutilización del medio natural, y consecuentemente su reproducción, lo más eficientemente posible para la promoción de sistemas de producción y consumo razonable (Roberts, 2004), así como la reducción de cantidades de residuos (materiales y emisiones) producidas para convertir subproductos en productos y recursos reutilizables. En este sentido, la ubicación e integración de empresas que puedan utilizar los residuos de los 
demás son cruciales. La SI puede ofrecer importantes beneficios económicos, ambientales y sociales, tanto a las empresas de forma individual como a una red de firmas (Desrochers, 2004).

Batten (2009) muestra, por medio de una simulación, la importancia de la SI para ayudar a identificar los posibles elementos de un ecosistema industrial, que pudieran trabajar juntos para lograr resultados más ecoeficientes y cómo se puede generar energía rentable futura en el que grupos de empresas se comporten más ecoeficientemente, introduciendo clusters estratégicamente ubicados de generación distribuida de bajo nivel de emisiones. Explica el rol que juega el modelado participativo y cómo puede construir la confianza y reducir el conflicto sobre la distribución del fondo común de recursos como agua y energía entre pequeños grupos de agentes en evolución.

\section{Argumentos EN CONTRA DE LA SI}

$\mathrm{L}$ as ineficiencias son inevitables debido a la pérdida de energía y materiales en diferentes puntos a lo largo del ciclo de producción/consumo/reciclaje/reutilización. Además, el modelado completo de sistemas industriales ecológicos con base en principios ecológicos biológicos es erróneo, puesto que los sistemas culturales y biológicos son fundamentalmente diferentes (Norton et al., 1998), asumiendo que el ciclo que se pretende cerrar se sustenta en sistemas culturales siempre fijos, o al menos con una constante de permanencia, lo que imposibilita la aplicación de la SI, es decir, si la experiencia muestra que las empresas prefieren hacerse cargo, a su manera, de los residuos, no es factible esperar un cambio de mentalidad en el corto o mediano plazos (Tudor et al., 2007).
Por otro lado, una limitación importante en el desarrollo de la SI es la "fragilidad” potencial del sistema. Una pequeña red industrial es vulnerable a una de las principales empresas que forman esta red, ya que al dejar o buscar en otros lugares sus materiales y productos puede afectar el funcionamiento de toda la cadena (Fernández, \& Ruiz, 2009). Existe una relación estrecha entre la SI, ciencia de sistemas complejos y el manejo de los recursos naturales. Todos ellos implican las interdependencias entre elementos de diversos tipos del sistema (Perry, \& Boon, 2004).

Los ecosistemas de nuestro planeta son inherentemente difíciles de manejar con éxito, debido a la complejidad e incertidumbre asociadas a su evolución (Andersen, 2007). Gran parte de esta complejidad e incertidumbre proviene de fuentes humanas (Sagoff, 1998). Por ejemplo, los agentes económicos del mercado o agentes sociales de la comunidad están continuamente haciendo decisiones acerca de si deben trabajar conjuntamente en forma competitiva o cooperativa y cómo. Lo que deciden tiene implicaciones importantes para la biósfera en la que todos vivimos.

\section{Conciliación}

e sabe que uno de los principales $\circlearrowleft$ enemigos de la civilización moderna, es la contaminación ambiental (Cote, \& Cohen-Rosenthal, 1998), que conduce a diversos desórdenes de la salud en los habitantes, así como a afectaciones serias en el entorno socioecológico (Tudor et al., 2007).

Las estrategias planteadas en este documento a favor de la SI, van encaminadas a minimizar este impacto ambiental; sin embargo, hay argumentos que marcan un contrasentido en la utilización de recursos naturales, dado que querer cerrar el círculo de la producción industrial y por ende, la reducción de contaminantes, hace muy frágil esta cadena de suministro, que, de romperse, crearía más caos que el beneficio que esto produce (Desrochers, 2004).

Y si bien la propuesta de utilizar estos desechos energéticos y materiales como subproductos de otras industrias dependerá de la estrategia que se tenga para mantener lo más cercano posible a estas empresas, una gran parte del éxito recae en la ampliación de nuestro conocimiento acerca de cómo y por qué elegimos interactuar entre sí y cómo esas decisiones colectivas afectan nuestra biósfera y sus ecosistemas con el paso del tiempo

\section{Conclusiones}

a implementación de un modelo
de SI no exige un gran esfuerzo
de planeación, basta con ubicar geográfica y estratégicamente a empresas que generen materia y energía excedente y que quieran compartir y/o recibir para sus propios procesos y el del cluster. En este intento, se habrá avanzado en el conocimiento y de la SI, y en la modificación de patrones culturales fijos en la industria.

El principal obstáculo que debe superarse, quizás el más importante, es que mientras los conceptos de EI han existido desde hace varias décadas, la "fabricación” en un modelo de SI no, y todavía necesita tiempo para ser una opción probada y sostenible (es decir, a largo plazo). Considerando que la mayoría de los parques industriales actuales han existido durante 10 o 15 años, es importante que ampliemos nuestro conocimiento sobre cómo y por qué interactuar con otras personas, porque de esta forma se pueden tomar decisiones tempranas que ayuden a nuestra biósfera y sus ecosistemas con 
el paso del tiempo, antes de optar por un modelo de SI.

\section{REFERENCIAS BIBLIOGRÁFICAS}

Andersen, M. S. (2007). An Introductory Note on the Environmental Economics of the Circular Economy. Sustain Sci (2), 133-140. Available from: http://dx.doi.org/110.1007/ s11625-11006-10013-11626

Batten, D. (2009). Fostering Industrial Symbiosis With Agent-Based Simulation and Participatory Modeling. Journal of Industrial Ecology, volumen 13, Issue: 2, pp. 197-213. ISSN: 1530-9290

Chiu, A., \& Yong, G. (2004). On the Industrial Ecology Potential in Asian Developing Countries. Journal of Cleaner Production (12), 1037-1045. Available from: http://dx.doi.org/1010.1016/j.jclepro.2004.1002.1013

Cohen-Rosenthal, E. (2004). Making sense out of industrial ecology: a framework for analysis and action. Journal of Cleaner Production, volumen: 12. Issue: 8. Pp. 1111-1123.

Cote, R. P., \& Cohen-Rosenthal, E. (1998). Designing Eco-industrial Parks: a Synthesis of Some Experiences. Journal of Cleaner Production (6), 181-188.

Desrochers, P. (2004). Industrial Symbiosis: the Case for Market Coordination. Journal of Cleaner Production (12), 1099-1101. Available from: http://dx.doi.org/1010.1016/j.jclepro.2004.1002.1008

Fernández, I., \& Ruiz, M. C. (2009). Descriptive Model and Evaluation System to Locate Sustainable Industrial Areas. Journal of Cleaner Production (17), 87-100. Available from: http://dx.doi.org/110.1016/j.jclepro.2008.1002.1011

Norton, B., Constanza, R., \& Bishop, R. (1998). The Evolution of Preferences Why "Sovereign" Preferences May not Lead to Sustainable Policies and What to Do About It. Ecological Economics (24), 193-211.

Perry, P.-J., \& Boon, O. (2004). Applying Ecosystem Concepts to the Planning of Industrial Areas: a Case Study of Singapore's Jurong Island. Journal of Cleaner Production (12), 1011-1023. Available from: http://doi.dx.org/1010.1016/j.jclepro.2004.1002.1028

Roberts, B. (2004). The Application of Industrial Ecology Principles and Planning Guidelines for the Development of Eco-industrial Parks: an Australian Case Study. Journal of Cleaner Production (12), 997-1010. Available from: http://dx.doi.org/1010.1016/j.jclepro.2004.1002.1037

Sagoff, M. (1998). Aggregation and Deliberation in Valuing Environmental Public Goods: a Look beyond Contingent Pricing. Ecological Econo$\operatorname{mics}(24), 213-230$.

Tudor, T., Adam, E., \& Bates, M. (2007). Drivers and Limitations for the Successful Development and Functioning of EIPs (Eco-industrial Parks): a Literature Review. Ecological Economics (61), 199-207. Available from:http://dx.doi.org/110.1016/j. ecolecon.2006.1010.1010 